\title{
Note on Qualitative Robustness of Multivariate Sample Mean and Median
}

\author{
Evgueni Gordienko, Andrey Novikov, and J. Ruiz de Chávez \\ Department of Mathematics, Autonomous Metropolitan University, Iztapalapa, San Rafael Atlixco 186, \\ Col. Vicentina, C.P. 09340, Mexico City, DF, Mexico
}

Correspondence should be addressed to J. Ruiz de Chávez; jrch@xanum.uam.mx

Received 4 October 2012; Revised 11 December 2012; Accepted 12 December 2012

Academic Editor: Shein-chung Chow

Copyright (C) 2013 Evgueni Gordienko et al. This is an open access article distributed under the Creative Commons Attribution License, which permits unrestricted use, distribution, and reproduction in any medium, provided the original work is properly cited.

\begin{abstract}
It is known that the robustness properties of estimators depend on the choice of a metric in the space of distributions. We introduce a version of Hampel's qualitative robustness that takes into account the $\sqrt{n}$-asymptotic normality of estimators in $R^{k}$, and examine such robustness of two standard location estimators in $\mathbb{R}^{k}$. For this purpose, we use certain combination of the Kantorovich and Zolotarev metrics rather than the usual Prokhorov type metric. This choice of the metric is explained by an intention to expose a (theoretical) situation where the robustness properties of sample mean and $L_{1}$-sample median are in reverse to the usual ones. Using the mentioned probability metrics we show the qualitative robustness of the sample multivariate mean and prove the inequality which provides a quantitative measure of robustness. On the other hand, we show that $L_{1}$-sample median could not be "qualitatively robust" with respect to the same distance between the distributions.
\end{abstract}

\section{Introduction}

The following Hampel's definition (originally given for the one-dimensional case) of qualitative robustness $[1,2]$ deals with $\pi$-balls in the space of distributions rather than with standard "contamination neighborhoods" (see for the latter, e.g., $[3,4])$.

The sequence $Q_{n}, n \geq 1$, of estimators is qualitatively robust at the distribution $\mathscr{L}$ if for every $\epsilon>0$ there exists $\delta>0$ such that $\pi(\mathscr{L}, \widetilde{\mathscr{L}})<\delta$ entails

$$
\sup _{n \geq 1}\left(Q_{n}\left(X_{1}, \ldots, X_{n}\right), Q_{n}\left(\widetilde{X}_{1}, \ldots, \widetilde{X}_{n}\right)\right)<\epsilon .
$$

Here, and throughout, $\pi$ denotes the Prokhorov metric, and $X_{1}, X_{2}, \ldots ; \widetilde{X}_{1}, \widetilde{X}_{2}, \ldots$, are i.i.d. random vectors distributed, respectively, as $\mathscr{L}$ and $\widetilde{\mathscr{L}}$.

For a metric $\mu$ on the space of distributions and random vectors $X, Y$ we will write $\mu(X, Y)$ (as in (1)) having in mind the $\mu$-distance between the distributions of $X$ and $Y$.

By all means, the use of the Prokhorov metric is only an option. For instance, in [5] other probability metrics in the definition of qualitative robustness were used. (See also [69] for using different probability metrics or pseudo-metrics related to the estimation of robustness).

As noted in $[1,2]$ in $\mathbb{R}^{1}$, sample means are not qualitatively robust at any $\mathscr{L}$, while sample medians are qualitatively robust at any $\mathscr{L}$ having a unique median. (See also [10] for lack of qualitative robustness of sample means in certain Banach spaces).

Moreover, in [11] it was shown that for symmetric distributions the median is, in certain sense, the "most robust" estimator of a center location when using the pseudo-metric corresponding to neighborhoods of contamination type. (See more with this respect in [8]).

At the same time, it is known from the literature that under different circumstances, in particular, using distinct probability metrics (as, e.g., in (1) or in other definitions) the robustness properties of estimators can change considerably (see, for instance, the discussion in [12]).

The first aim of the present paper is to consider a modified version of Hampel's definition of qualitative robustness taking into account the $\sqrt{n}$-asymptotic normality of the sequence of 
estimators. The formal definition is given in the next section but, basically, we replace (1) with the following condition:

$$
\sup _{n \geq 1} \sqrt{n} l\left(Q_{n}\left(X_{1}, \ldots, X_{n}\right), Q_{n}\left(\widetilde{X}_{1}, \ldots, \widetilde{X}_{n}\right)+\gamma\right) \leq \varepsilon,
$$

where $\gamma$ is some constant, and $l$ is a probability metric (different from the Prokhorov metric in our case).

The second goal of the paper is to present an example of two probability metrics $l$ and $\mu$ (on the space of distributions in $\mathbb{R}^{k}$ ) for which the following holds:

(i) when $Q_{n}=T_{n}, n \geq 1$, are multivariate sample means, the left-hand side of inequality (2) (with some $\gamma$ ) is bounded by const $\cdot \mu(\mathscr{L}, \widetilde{\mathscr{L}})$;

(ii) when $Q_{n}=M_{n}, n \geq 1$, are sample medians, we give an example of symmetric smooth distributions $\mathscr{L}$ and $\widetilde{\mathscr{L}}_{\alpha}, \alpha>0$, in $\mathbb{R}$, such that $\mu\left(\mathscr{L}, \widetilde{\mathscr{L}}_{\alpha}\right) \rightarrow 0$ as $\alpha \rightarrow 0$, while there is a positive constant $r$ such that the lefthand side of inequality (2) (with $\gamma=0$ ) is greater than $r$ for all sufficiently small $\alpha>0$. Therefore, sample medians are not qualitative robust (in our sense) with respect to these metrics.

The metrics $l$ and $\mu$ are the following (the complete definitions are given in Section 2). $l$ is the Kantorovich metric (see, e.g., [13]) and $\mu$ is certain combination of $l$ and of the Zolotarev metric of order 2 (see [14]).

We should stress that this choice is not determined by any advantages for statistical applications. Moreover, the closeness of distributions in the Kantorovich metric implies the closeness in the Prokhorov metric, but also reduces the probability of "large-valued outliers" (but not the rounding errors). Therefore, our selection of metric is not quite consistent with the standard approach to qualitative robustness where the Prokhorov metric (or its invariant versions) is used.

Nevertheless, our choice allows to unveil the possible unusual robustness properties of sample means and medians and to assess the certain quantitative robustness of the multivariate sample mean (with respect to the considered metrics!). The obtained "robustness inequality" does not work in the "gross error model" but (jointly with inequalities (23)) it could be useful for quantitative assessment of the robustness of sample means, under perturbation of data of "rounding" type.

\section{Basic Definitions}

Let $(H,|\cdot|)$ be a separable Hilbert space with the norm $\mid$. | generated by an inner product $\langle\cdot, \cdot\rangle$, and let $\mathscr{B}(H)$ be the Borel $\sigma$-algebra of subsets of $H$. Let also $X ; X_{1}, \ldots, X_{n}, \ldots$ and $\widetilde{X} ; \widetilde{X}_{1}, \ldots, \widetilde{X}_{n}, \ldots$ be two sequences of i.i.d. random vectors in $H$ with their respective distributions denoted by $\mathscr{L}$ and $\widetilde{\mathscr{L}}$.

Under the assumption

$$
E|X|<\infty, \quad E|\widetilde{X}|<\infty,
$$

the means $E X$ and $E \widetilde{X}$ are defined as the corresponding Bochner integrals.
The definition of the median of $X$ is less standard (consult, e.g., [15] for different definitions in $H=\mathbb{R}^{k}$ ). We will use the definition of median given in general setting in [16] (sometimes called $\mathbb{L}_{1}$-median):

$$
\begin{gathered}
M:=\underset{x \in H}{\arg \min } E(|X-x|-|x|), \\
\widetilde{M}:=\underset{x \in H}{\arg \min } E(|\widetilde{X}-x|-|x|) .
\end{gathered}
$$

In [16] it was shown that $M$ exists, and it is unique unless $\mathscr{L}$ is concentrated on a one-dimensional subspace of $H$. In the last case the set of minimizers in (4) is $\left\{t x_{0}: t \in[a, b]\right\}$ for some $x_{0} \in H$, and one can set $M:=((a+b) / 2) x_{0}$.

In what follows we denote $(n=1,2, \ldots)$ :

$$
\begin{gathered}
S_{n}:=X_{1}+\cdots+X_{n}, \quad \widetilde{S}_{n}:=\widetilde{X}_{1}+\cdots+\widetilde{X}_{n}, \\
T_{n}=\frac{S_{n}}{n}, \quad \widetilde{T}_{n}:=\frac{\widetilde{S}_{n}}{n} \quad \text { (sample means). }
\end{gathered}
$$

On the other hand, let $(n=1,2, \ldots)$,

$$
M_{n}=M_{n}\left(X_{1}, \ldots, X_{n}\right), \quad \widetilde{M}_{n}=\widetilde{M}_{n}\left(\widetilde{X}_{1}, \ldots, \widetilde{X}_{n}\right)
$$

be sample medians defined by (4) and (5) replacing $\mathscr{L}$ and $\widetilde{\mathscr{L}}$ by the corresponding empirical distributions $\mathscr{L}_{n}$ and $\widetilde{\mathscr{L}}_{n}$ (obtained from $X_{1}, \ldots, X_{n}$ and $\widetilde{X}_{1}, \ldots, \widetilde{X}_{n}$, resp.). Robustness (in terms of $\epsilon$-contamination neighborhoods) and asymptotic normality of $M_{n}, n \geq 1$, were proved, for instance, in [17] (see also [18]). The qualitative robustness properties of sample means $\left\{T_{n}\right\}$ and sample medians $\left\{M_{n}\right\}$ when the Prokhorov metric is used were discussed in Introduction.

Let us first see what happens with qualitative robustness of $\left\{T_{n}\right\}$ and $\left\{M_{n}\right\}$ if in the above definition we replace $\pi$ with the Kantorovich metric:

$$
l(X, \widetilde{X}) \equiv l(\mathscr{L}, \widetilde{\mathscr{L}}):=\sup _{\phi \in \operatorname{Lip}}|E \phi(X)-E \phi(\widetilde{X})|,
$$

where

$$
\begin{aligned}
& \text { Lip }:=\{\phi: H \longmapsto \mathbb{R}: \phi \text { is bounded and } \\
&|\phi(x)-\phi(y)| \leq|x-y| ; x, y \in H\} .
\end{aligned}
$$

Under condition (3) $l(X, \widetilde{X})<\infty$, and it is well known (see, e.g., [13]) that $l\left(Y_{n}, Y\right) \rightarrow 0$ if and only if $\pi\left(Y_{n}, Y\right) \rightarrow 0$ and $E\left|Y_{n}\right| \rightarrow E|Y|$.

For $H=\mathbb{R}$,

$$
l(X, \widetilde{X})=\int_{-\infty}^{\infty}\left|F_{X}(x)-F_{\widetilde{X}}(x)\right| d x .
$$
[13]):

Now, applying the regularity properties of $l$ (see, e.g.,

$$
l\left(\frac{S_{n}}{n}, \frac{\widetilde{S}_{n}}{n}\right) \leq \frac{1}{n} \sum_{i=1}^{n} l\left(X_{i}, \widetilde{X}_{i}\right)=l(\mathscr{L}, \widetilde{\mathscr{L}}),
$$

we see that the sequence of sample means $\left\{T_{n}, n \geq 1\right\}$ is "qualitatively robust" using $l$ instead of the Prokhorov metric. 
It seems straightforward (using the approach similar to one given in $[10,19]$ and results in [16]) to show that the sequence of sample medians $\left\{M_{n}, n \geq 1\right\}$ is also "qualitatively robust" with respect to $l$. However, this is out of the scope of this paper.

\section{3. $l-\mu-\sqrt{n}$-Robustness and Main Results}

Now and in what follows we suppose that $H=\mathbb{R}^{k}$ with the Euclidean norm $|\cdot|$. The results presented in this section are an extension to the multidimensional case of the similar findings for $H=\mathbb{R}$, published in the hardly accessible proceedings [20]. Moreover, we improve the results of [20] even in the onedimensional case.

In order to simplify calculations in the proof of Theorem 5 below we will use in the definitions of Kantorovich's and Zolotarev's metrics (see below) the following norm $\|x\|:=$ $\sum_{i=1}^{k}\left|x_{i}\right|$ in the space $\mathbb{R}^{k}$.

Thus, in what follows the Kantorovich metric $l$ is defined by relationships (8), (9), where in (9) in place of the norm $|\cdot|$ the norm $\|\cdot\|$ is used.

Let $\mu$ be some fixed simple probability metric on the set of all probability distributions on $\left(\mathbb{R}^{k}, \mathscr{B}\left(\mathbb{R}^{k}\right)\right)$.

We will consider a sequence $Q_{n}, n \geq 1$, of estimators of some parameter $\theta \in \mathbb{R}^{k}$ of the distribution $\mathscr{L}(\widetilde{\theta}$ of the distribution $\widetilde{\mathscr{L}}$, resp.).

Definition 1. We say that a sequence $Q_{n}, n \geq 1$, is $(l-\mu-$ $\sqrt{n}$ )-robust at $\mathscr{L}$ if there is some fixed vector $\gamma=\gamma(\mathscr{L}, \widetilde{\mathscr{L}}) \epsilon$ $\mathbb{R}^{k}$ such that for every $\epsilon>0$ there exists $\delta>0$ such that $\mu(\mathscr{L}, \widetilde{\mathscr{L}}) \leq \delta$ entails

$$
\sup _{n \geq 1} \sqrt{n} l\left(Q_{n}\left(X_{1}, \ldots, X_{n}\right), Q_{n}\left(\widetilde{X}_{1}, \ldots, \widetilde{X}_{n}\right)+\gamma\right) \leq \epsilon .
$$

Remark 2. Taking into account that $l(a X+b, a Y+b)=$ al $(X, Y), a \geq 0, b \in \mathbb{R}^{k}$, we see that (12) can be related with the $\sqrt{n}$-asymptotic normality of estimators $Q_{n}, n \geq 1$. The "scaling parameter" $\gamma$ (in (12)) is necessary to ensure the equality of means of the corresponding limit normal distributions (when they exist). Only in case $E X=E \widetilde{X}$ in (12) $\gamma=0$.

Zolotarev's probability metric of order $2 \zeta_{2}$ is defined as follows (see, e.g., [14, 21]):

$$
\zeta_{2}(X, \widetilde{X}) \equiv \zeta_{2}(\mathscr{L}, \widetilde{\mathscr{L}}):=\sup _{\phi \in \mathscr{D}_{2}}|E \phi(X)-E \phi(\widetilde{X})|,
$$

where

$$
\begin{gathered}
\mathscr{D}_{2}:=\left\{\phi: \mathbb{R}^{k} \longmapsto \mathbb{R}:\|D \phi(x)-D \phi(y)\| \leq\|x-y\| ;\right. \\
\left.x, y \in \mathbb{R}^{k}\right\},
\end{gathered}
$$

and $D \phi=\left(D_{1} \phi, \ldots, D_{k} \phi\right)$ is the gradient of $\phi$.

Remark 3. The distance $\zeta_{2}(X, \widetilde{X})$ can take infinite value. Particularly, from (13), (14) we see that $\zeta_{2}(X, \widetilde{X})=\infty$ if
$E X \neq E \widetilde{X}$. On the other hand, if there exist second moments, $E X=E \widetilde{X}$ and $E X^{2}<\infty, E \widetilde{X}^{2}<\infty$, then $\zeta_{2}(X, \widetilde{X})<\infty$ (see, e.g., [14]). The function $\phi(x):=(1 / 2)|x|^{2} \in \mathscr{D}_{2}$ in (14). Therefore, by (13),

$$
\left|E X^{2}-E \widetilde{X}^{2}\right| \leq \zeta_{2}(X, \widetilde{X}) .
$$

Thus, if $E X^{2}<\infty$ and $\zeta_{2}(X, \widetilde{X})<\infty$ then $E \widetilde{X}^{2}<\infty$. Consequently, if $E X=E \widetilde{X}$ and $E|X|^{2}<\infty$, then $\zeta_{2}(X, \widetilde{X})<$ $\infty$ if and only if $E|\widetilde{X}|^{2}<\infty$.

We now define the metric $\mu$ to work with:

$$
\mu(X, \widetilde{X}):=\max \left\{2 l(X, \widetilde{X}), \inf _{b \in \mathbb{R}^{k}} \zeta_{2}(X, \widetilde{X}+b)\right\} .
$$

3.1. $(l-\mu-\sqrt{n})$-Robustness of Sample Means. To prove the inequality in Theorem 5 below we need to impose the following restriction on the distribution $\mathscr{L}$.

Assumption 4. (i) $E|X|^{k+2}<\infty$, and the covariance matrix $M$ of $X$ is positive definite.

The distribution $\mathscr{L}$ of $X$ has a density $f$ such that for some $s \geq 1:$

(ii) the density $f_{s}$ of $S_{s}:=X_{1}+\cdots+X_{s}$ is bounded and differentiable;

(iii) the gradient $D f_{s}$ is bounded and $\left|D f_{s}\right|$ belongs to $\mathbb{L}_{1}\left(\mathbb{R}^{k}\right)$;

(iv) for some $\alpha>0$

$$
\int_{|x|>\alpha n}\left|D f_{s}\right| d x=O\left(n^{-1 / 2}\right), \quad \text { as } n \longrightarrow \infty .
$$

Note that in view of Remark 3 under the condition (i) $\mu(X, \widetilde{X})<\infty$ if and only if

$$
E|\widetilde{X}|^{2}<\infty
$$

(see (15)).

Theorem 5. Under Assumption 4 and supposing (18) it holds

$$
\sup _{n \geq 1} \sqrt{n} l\left(T_{n}, \widetilde{T}_{n}+\gamma\right) \leq c \mu(\mathscr{L}, \widetilde{\mathscr{L}}),
$$

where $\gamma=E X-E \widetilde{X}$,

$$
\begin{aligned}
& c=\max \left\{(10 s-1)^{1 / 2}, 5.4 d k\right\}, \\
& d=\sup _{n \geq s} \max _{1 \leq i \leq k}\left\|D_{i} g_{n}\right\|_{\mathbb{L}_{1}\left(\mathbb{R}^{k}\right)}<\infty,
\end{aligned}
$$

and $g_{n}$ is the density of $(1 / \sqrt{n})\left(X_{1}+\cdots+X_{n}\right), n \geq 1$.

Remark 6. (i) The constant $c$ in (20), (21) is entirely determined by the distribution $\mathscr{L}$ of $X$. For various particular densities of $X$ the constant $d$ in (21) can be bounded by means of computer calculations. For this one can use the fact (true under wide conditions) that the sequence $D_{i} g_{n}, n \geq s$, converges in $\mathbb{L}_{1}$-norm to the corresponding partial derivative 
of the limit normal density with covariance matrix $\mathscr{M}$ (and zero mean since $\left\|D_{i} g_{n}\right\|_{\mathbb{L}_{1}\left(\mathbb{R}^{k}\right)}$ is invariant under translations).

For example, let $k=2$ and $X=\left(X^{\prime}, X^{\prime \prime}\right)$, where $X^{\prime}$ and $X^{\prime \prime}$ are independent random variables; $X^{\prime}$ has the gamma density with $\alpha=2.1$ and arbitrary $\lambda^{\prime}>0$, while $X^{\prime \prime}$ has the gamma density with $\alpha=3$ and arbitrary $\lambda^{\prime \prime}$. Simple computer calculations show that in $(21) d<\max \left\{0.7065 \lambda^{\prime}, 0.5414 \lambda^{\prime \prime}\right\}$, and since we can take $s=1$, we obtain in (20) that

$$
c<\max \left\{3,10.8 \max \left\{0.7065 \lambda^{\prime}, 0.5414 \lambda^{\prime \prime}\right\}\right\} .
$$

For instance, $c<7.6302$ for $\lambda^{\prime}=\lambda^{\prime \prime}=1$. (For these values of $\lambda^{\prime}, \lambda^{\prime \prime}$ we can take $s=3$ in (20) and obtain $c<6.3829$.)

(ii) Since under the above assumption $\mu(\mathscr{L}, \widetilde{\mathscr{L}})<\infty$ entails (18), inequality (19) ensures $(l-\mu-\sqrt{n})$-robustness of the sequence of sample means $T_{n}, n \geq 1$.

(iii) For $k=1$, in [20] an example is given showing that in general the sequence of sample means $T_{n}, n \geq 1$ is not $(l-l-\sqrt{n})$-robust (even if (18) holds and $E X=E \widetilde{X})$. It is also almost evident that the sample means $T_{n}, n \geq 1$, are not $(l-\mu-\sqrt{n})$-robust, for example, if $\mu$ is the total variation metric $V$ (or, if $\mu=\max (l, V)$ ). The appearance of Zolotarev's metric on the right-hand side of (19) is related to closeness of corresponding limit normal distributions.

Corollary 7. Suppose for a moment that $\theta=\tilde{\theta}$ and that one evaluates the quality of estimators by mean of absolute errors: $\delta_{n}:=E\left\|T_{n}-\theta\right\|, \widetilde{\delta}_{n}:=E\left\|\widetilde{T}_{n}-\theta\right\|$. Then from (19) it follows that

$$
\left|\delta_{n}-\widetilde{\delta}_{n}\right| \leq n^{-1 / 2} \text { const } \mu(\mathscr{L}, \widetilde{\mathscr{L}}), \quad n \geq 1
$$

(The simple proof is similar to the one given in [20]).

3.2. About $(l-\mu-\sqrt{n})$-Robustness of Sample Medians. Let again $\mu$ be the metric defined in (16). We show that the sequence of sample medians $M_{n}, n \geq 1$, in general, is not $(l-\mu-\sqrt{n})$-robust even when $X$ and $\widetilde{X}$ have strictly positive, bounded, smooth densities symmetric with respect to the origin, and the sequences of sample medians $M_{n}, n \geq 1, \widetilde{M}_{n}$, $n \geq 1$, are $\sqrt{n}$-asymptotically normal. We consider a modified version of the corresponding example from [20].

Example 8. Let $H=\mathbb{R}, X \sim N(0,1)$, and for $\epsilon \in(0,1)$ let $\widetilde{X}_{\epsilon}$ be a random variable with the density:

$$
f_{\widetilde{X}_{\epsilon}}(x):=\frac{1}{c(\epsilon)}\left(\epsilon+\frac{x^{2}}{\epsilon^{3}+x^{2}}\right) e^{-x^{2} / 2}, \quad x \in \mathbb{R},
$$

where $c(\epsilon)$ is a normalizing constant. By symmetry of the density, we get

$$
M=\widetilde{M}=E X=E \widetilde{X}=0
$$

and also it is clear that $f_{X}, f_{\widetilde{X}} \in \mathbb{C}^{\infty}(\mathbb{R})$, and

$$
\sup _{\epsilon \in(0,1), x \in \mathbb{R}} f_{\widetilde{X}_{\epsilon}}(x)<\infty
$$

First of all let us show that for this example the left-hand side of (12) is infinite for any $\gamma \neq 0$. It is well known (see, e.g., [16]) that $M_{n} \rightarrow M=0, \widetilde{M}_{n} \rightarrow \widetilde{M}=0$ as $n \rightarrow \infty$ with probability 1 . Also, from the results of [16] we can obtain that $\left|M_{n}\right| \leq(2 / n) \sum_{i=1}^{n}\left|X_{i}\right|$. Using this inequality it is easy to show that the sequence $\left|M_{n}\right|, n \geq 1$, is uniformly integrable. Therefore, $E\left|M_{n}\right| \rightarrow 0$ (and also $E\left|\widetilde{M}_{n}\right| \rightarrow 0$ ) as $n \rightarrow \infty$, and for this $l\left(M_{n}, \widetilde{M}_{n}+\gamma\right) \rightarrow l(0, \gamma)=\gamma$.

Let now $\gamma=0$ in (12). By the well-known asymptotic normality (see [22, page 307]),

$$
\pi\left(\sqrt{n} M_{n}, \eta\right) \longrightarrow 0, \quad \pi\left(\sqrt{n} \widetilde{M}_{n}, \eta_{\epsilon}\right) \longrightarrow 0,
$$

where

$$
\begin{gathered}
\eta \sim N\left(0, \frac{1}{2 f_{X}(0)}\right)=N\left(0, \sqrt{\frac{\pi}{2}}\right), \\
\eta_{\epsilon} \sim N\left(0, \frac{1}{2 f_{\widetilde{X}_{\epsilon}}(0)}\right)=N\left(0, \frac{c(\epsilon)}{\epsilon}\right) .
\end{gathered}
$$

From (27) and (28) it follows that there is $r>0$ such that for all small enough $\epsilon$ and all large enough $n$ we get $\pi\left(\sqrt{n} M_{n}, \sqrt{n} \widetilde{M}_{n}\right) \geq r$. Consequently, for all such $\epsilon$ and $n$,

$$
\sqrt{n} l\left(M_{n}, \widetilde{M}_{n}\right)=l\left(\sqrt{n} M_{n}, \sqrt{n} \widetilde{M}_{n}\right) \geq r^{2}>0,
$$

since $l \geq \pi^{2}$ (see [13, page 86$\left.]\right)$.

We have obtained that the left-hand side of (12) is positive for all small enough $\epsilon>0$. On the other hand, $\mu\left(X, \widetilde{X}_{\epsilon}\right) \rightarrow 0$ as $\epsilon \rightarrow 0$. It follows from the inequality

$$
\zeta_{2}(X, Y) \leq \frac{1}{2} \int_{-\infty}^{\infty} x^{2}\left|f_{X}(x)-f_{Y}(x)\right| d x
$$

(valued when $E X=E Y$; see [21, page 376]) and from (10).

Remark 9. The densities as in (24) represent the following somewhat strange type of "contamination." Since $\max _{x} f_{X}(x)=f_{X}(0)$ sample points from $f_{X}$ tend to concentrate around the origin. But $f_{\widetilde{X}_{\epsilon}}(0) \rightarrow 0$ as $\epsilon \rightarrow 0$, and therefore sample points from $f_{\widetilde{X}_{\epsilon}}$ frequently in some extent are separated from 0 .

A natural question is "how to choose the metric $\tilde{\mu}$ to ensure $(l-\tilde{\mu}-\sqrt{n})$-robustness of the sequence of sample medians $M_{n}, n \geq 1$ ?" Our conjecture is (for $k=1$, e.g.) to try $\widetilde{\mu}(X, \widetilde{X})=\max \left\{l(X, \widetilde{X})\right.$, $\left.\operatorname{esssup}_{x \in \mathbb{R}}\left|f_{X}(x)-f_{\widetilde{X}}(x)\right|\right\}$ (supposing the existence of densities).

If $M=\widetilde{M}$ then under certain conditions the closeness in $\tilde{\mu}$ guarantees the closeness of normal densities which are limiting for $\left\{\sqrt{n} M_{n}, n \geq 1\right\}$ and for $\left\{\sqrt{n} \widetilde{M}_{n}, n \geq 1\right\}$, respectively. To attempt proving $(l-\tilde{\mu}-\sqrt{n})$-robustness of $M_{n}, n \geq 1$ (as in (12)) one can show Hampel's qualitative robustness of $M_{n}, n \geq 1$ with respect to the metric $l$ and then use the property $\sqrt{n} l\left(M_{n}, \widetilde{M}_{n}\right)=l\left(\sqrt{n} M_{n}, \sqrt{n} \widetilde{M}_{n}\right)$. A not clear point of this plan is finding conditions under which $E\left|\sqrt{n}\left(M_{n}-M\right)\right| \rightarrow 0$ as $n \rightarrow \infty$. 
Example 10. Let us give another (very simple) example of the sequence of estimators which is $(l-\mu-\sqrt{n})$-robust with $\mu:=l$ (on the class of distributions described below). For $H=\mathbb{R}$ we consider the class $W$ of all random variables $X$ with bounded supports $\operatorname{Supp}(X)=\left[0, \theta_{X}\right]$, having density $f_{X}$ such that $f_{X}(x) \geq \beta>0, x \in\left[0, \theta_{X}\right]$. We suppose that $\theta_{X} \leq \theta^{*}<\infty, X \in W$, and $\beta$ is the same for all $X \in W$. Assume that parameter $\theta=\theta_{X}$ is unknown, and the sequence of estimators $Q_{n}:=\max _{1 \leq i \leq n} X_{i}$ is used to estimate it.

Denoting $\theta=\theta_{X}, \widetilde{\theta}=\widetilde{\theta}_{X}$ and choosing in (12) $\gamma=\theta-\widetilde{\theta}$, we get

$$
\begin{aligned}
l\left(Q_{n}, \widetilde{Q}_{n}+\gamma\right) & =l\left(\theta-\max _{1 \leq i \leq n} X_{i}, \widetilde{\theta}-\max _{1 \leq i \leq n} \widetilde{X}_{i}\right) \\
& \leq E\left|\left(\theta-\max _{1 \leq i \leq n} X_{i}\right)-\left(\widetilde{\theta}-\max _{1 \leq i \leq n} \widetilde{X}_{i}\right)\right| \\
& \leq E\left(\theta-\max _{1 \leq i \leq n} X_{i}\right)+E\left(\widetilde{\theta}-\max _{1 \leq i \leq n} \widetilde{X}_{i}\right),
\end{aligned}
$$

because of the metric $l$ is minimal for the compound metric $E|X-Y|$ (see, e.g., [13]).

By elementary calculations we bound the right-hand side of (31) by

$$
\frac{2}{\beta(n+1)} \text {. }
$$

Now for each $n$ fixed, by induction we obtain (see (10))

$$
\begin{aligned}
l\left(\max _{1 \leq i \leq n} X_{i}, \max _{1 \leq i \leq n} \widetilde{X}_{i}\right) & =\int_{0}^{\infty}\left|F_{X}^{n}(x)-F_{\widetilde{X}}^{n}(x)\right| d x \\
& \leq n \int_{0}^{\infty}\left|F_{X}(x)-F_{\widetilde{X}}(x)\right| d x=n l(X, \widetilde{X}) .
\end{aligned}
$$

On the other hand, let, for example, $\theta \leq \widetilde{\theta}$. Then

$$
l(X, \widetilde{X})=\int_{0}^{\theta}\left|F_{X}(x)-F_{\widetilde{X}}(x)\right| d x+\int_{\theta}^{\tilde{\theta}}\left(1-F_{\widetilde{X}}(x)\right) d x
$$

But for $x \in[\theta, \widetilde{\theta}]$,

$$
1-F_{\widetilde{X}}(x)=\int_{x}^{\widetilde{\theta}} f_{\widetilde{X}}(y) d y \geq \beta(\widetilde{\theta}-x) .
$$

From this relationship it follows that

$$
\text { for } X, \widetilde{X} \in W, \quad \widetilde{\theta} \longrightarrow \theta \text { as } l(X, \widetilde{X}) \longrightarrow 0 .
$$

Finally, applying (32), we can select $N$ in such a way that

$$
\sup _{n \geq N} \sqrt{n} l\left(Q_{n}, \widetilde{Q}_{n}+\gamma\right) \leq \frac{\epsilon}{2} .
$$

For $n<N$ we can use the inequality $l(X, Y+b) \leq l(X, Y)+|b|$ and (33), (36). Thus, choosing small enough $\delta>0$ we can ensure inequality (12) for all $X, \widetilde{X} \in W$ with $\mu(X, \widetilde{X}) \equiv$ $l(X, \widetilde{X}) \leq \delta$. In this way we proved $(l-l-\sqrt{n})$-robustness of $Q_{n}$ on the set $W$.

\section{Appendix}

\section{A. The Proofs}

To start with the proof of Theorem 5 of the previous section, first of all we note that in (19)

$$
\begin{aligned}
l\left(T_{n}, \widetilde{T}_{n}+\gamma\right) & =l\left(\frac{S_{n}}{n}, \frac{\widetilde{S}_{n}}{n}+E X-E \widetilde{X}\right) \\
& =l\left(\frac{S_{n}-n E X}{n}, \frac{\widetilde{S}_{n}-n E \widetilde{X}}{n}\right),
\end{aligned}
$$

because of $l(X+b, Y+b)=l(X, Y), b \in \mathbb{R}^{k}$. Also it is easy to see that $\zeta_{2}(X+b, Y+b)=\zeta_{2}(X, Y)$.

On the other hand, by definition of $\mu$ in (16)

$$
\begin{aligned}
\inf _{b \in \mathbb{R}^{k}} \zeta_{2}(X, \widetilde{X}+b) & =\zeta_{2}(X, \widetilde{X}+E X-E \widetilde{X}) \\
& =\zeta_{2}(X-E X, \widetilde{X}-E \widetilde{X})
\end{aligned}
$$

(see Remark 3). Also

$$
\begin{aligned}
l(X-E X, \widetilde{X}-E \widetilde{X}) & =l(X, \widetilde{X}+E X-E \widetilde{X}) \\
& \leq l(X, \widetilde{X})+E|X-\widetilde{X}| \leq 2 l(X, \widetilde{X}),
\end{aligned}
$$

because the metric $l$ is minimal for the metric $E|\cdot|$.

The above arguments show that to establish inequality (19) it suffices to prove the version of (19) with $\gamma=0, E X=$ $E \widetilde{X}=0$, and the metric $\mu^{\prime}:=\max \left\{l, \zeta_{2}\right\}$ on the right-hand side of (19).

The proof is based on the two following lemmas.

Let $g: \mathbb{R}^{k} \mapsto \mathbb{R}$ be a differentiable function. We will write $D g:=\left(D_{1} g, \ldots, D_{k} g\right)$, where $D_{i} g(x):=\left(\partial / \partial x_{i}\right) g(x)$, $i=1,2, \ldots, k$.

Lemma A.1. Let $X, Y$, and $\xi$ be random vectors in $\mathbb{R}^{k}$ such that

(a) $\xi$ is independent of $X$ and $Y$;

(b) $E|X|^{2}<\infty, E|Y|^{2}<\infty ; E X=E Y$;

(c) $\xi$ has a bounded differentiable density $f_{\xi}$ (with respect to the Lebesgue measure) such that $D_{i} f_{\xi} \in \mathbb{L}_{1}\left(\mathbb{R}^{k}\right), i=$ $1,2, \ldots, k$.

Then

$$
l(X+\xi, Y+\xi) \leq k \max _{1 \leq i \leq k}\left\|D_{i} f_{\xi}\right\|_{\mathbb{L}_{1}\left(\mathbb{R}^{k}\right)} \zeta_{2}(X, Y) .
$$

Proof. From the definition (8), (9) of the metric $l$ (with the norm ||$\cdot||$ instead of $|\cdot|$ !) it follows (the proof is simple) that in (8) the class of functions Lip (given in (9)) can be replaced by the class of all bounded differentiable functions $g: \mathbb{R}^{k} \mapsto \mathbb{R}$ such that

$$
\sup _{x \in \mathbb{R}^{k}}\left|\frac{\partial g(x)}{\partial x_{i}}\right| \leq 1, \quad i=1,2, \ldots, k
$$


Let us fix any such function $g$ and arbitrary $i, j \in$ $\{1,2, \ldots, k\}$. Then (by the Fubini theorem),

$$
\begin{aligned}
E g(X+\xi)-E g(Y+\xi) & \\
= & \int_{\mathbb{R}^{k}} g(x) d x \int_{\mathbb{R}^{k}} f_{\xi}(x-t)\left[d F_{X}(t)-d F_{Y}(t)\right] \\
& \times \int_{\mathbb{R}^{k}}\left[d F_{X}(t)-d F_{Y}(t)\right] \int_{\mathbb{R}^{k}} g(x) f_{\xi}(x-t) d x .
\end{aligned}
$$

For each $x \in \mathbb{R}$ fixed let

$$
\begin{aligned}
h(t) & :=\int_{\mathbb{R}^{k}} g(x) f_{\xi}(x-t) d x \\
& =\int_{\mathbb{R}^{k}} g(y+t) f_{\xi}(y) d y, \quad t \in \mathbb{R}^{k} .
\end{aligned}
$$

Because of boundedness of $\partial g / \partial t_{i}$ and integrability of $D_{i} f_{\xi}$ we can differentiate in (A.7) under the integral sign (see [23, Appendix A]). Thus,

$$
\begin{gathered}
\frac{\partial h}{\partial t_{j}} h(t)=\int_{\mathbb{R}^{k}} \frac{\partial g}{\partial t_{j}} g(y+t) f_{\xi}(y) d y, \\
\frac{\partial^{2} h}{\partial t_{i} \partial t_{j}} h(t)=-\int_{\mathbb{R}^{k}} \frac{\partial g}{\partial x_{j}}(x) \frac{\partial f_{\xi}}{\partial t_{i}}(x-t) d x .
\end{gathered}
$$

In view of (A.5) we obtain

$$
\left|\frac{\partial^{2} h}{\partial t_{i} \partial t_{j}} h(t)\right| \leq \int_{\mathbb{R}^{k}}\left|\frac{\partial f_{\xi}}{\partial t_{i}}(x-t)\right| d x=\left\|D_{i} f_{\xi}\right\|_{\mathbb{L}_{1}\left(\mathbb{R}^{k}\right)^{*}}
$$

For every $x, y \in \mathbb{R}^{k}$ we have

$$
\begin{gathered}
\|D h(x)-D h(y)\|=\sum_{j=1}^{k}\left|D_{j} h(x)-D_{j} h(y)\right|, \\
\left|D_{j} h(x)-D_{j} h(y)\right| \leq \sum_{i=1}^{k} \sup _{x \in \mathbb{R}^{k}} \frac{\partial^{2} h}{\partial x_{i} \partial x_{j}}(x)\left\|x_{i}-y_{i}\right\| .
\end{gathered}
$$

Comparing (A.6)-(A.11) and taking into account the definition of $\zeta_{2}$ in (13), (14), we obtain inequality (A.4).

Lemma A.2. Under the assumption of the previous section the constant $d$ in (21) is finite.

Remark A.3. A similar assertion was proved in [24] for $\|$. $\|_{\mathbb{L}_{1}}$-norm of second derivatives of the densities $g_{n}$ (under sightly different conditions). For this reason we give only a sketch of the proof of Lemma A.2 indicating only differences in comparison to the proof of Lemma 4.1 in [24] (where the omitted details can be seen).

Proof. As before, let $g_{n}(n \geq s)$ be the density of $S_{n} / \sqrt{n}$, and $\phi_{n}$ its characteristic function. Let also $f_{n}$ denote the density of $S_{n}$. There is $l \geq s$ such that $|t| \phi_{l}(t) \in \mathbb{L}_{1}\left(\mathbb{R}^{k}\right)$ and therefore

$$
|t| \phi_{n}(t) \in \mathbb{R}_{1}\left(\mathbb{R}^{k}\right), \quad \text { for } n \geq l .
$$

Indeed, since

$$
v_{i}\left(x_{i}\right):=\frac{\partial}{\partial x_{i}} f_{s}\left(\ldots, x_{i}, \ldots\right) \in \mathbb{L}_{1}(\mathbb{R})
$$

(fixing other variables), we can integrate by parts:

$$
\begin{aligned}
\phi_{s}(\sqrt{s} t) & =\int_{\mathbb{R}^{k-1}} e^{i\left\langle t^{\prime}, x^{\prime}\right\rangle} d x^{\prime} \int_{\mathbb{R}} f_{s}\left(\ldots, x_{i}, \ldots\right) e^{i t_{i} x_{i}} d x \\
& =\int_{\mathbb{R}^{k-1}} e^{i\left\langle t^{\prime}, x^{\prime}\right\rangle} d x^{\prime} \frac{1}{i t_{i}} \int_{\mathbb{R}} v_{i}\left(x_{i}\right) e^{i t_{i} x_{i}} d x_{i},
\end{aligned}
$$

and (A.12) follows for large enough $l$.

In view of (A.12) we can write down the inverse Fourier transform for $n \geq l$ :

$$
g_{n}(x)=\frac{1}{(2 \pi)^{k}} \int_{\mathbb{R}^{k}} \phi_{n}(t) e^{-i\langle t, x\rangle} d t,
$$

and differentiate under the integral sign in (A.15).

The condition $\phi_{n} \in \mathbb{L}_{1}\left(\mathbb{R}^{k}\right)(n \geq l)$ and Assumption (i) in Section 2 ensure the hypothesis of in [25, Theorem 19.2, Ch. 4]. By this theorem,

$$
g_{n}(x)=\sum_{m=0}^{k} n^{-m / 2} P_{m}(x) \phi_{0, \mathscr{M}}(x)+o\left(\frac{1}{n^{k / 2}}\right),
$$

$x \in \mathbb{R}^{k}$ (as $\left.n \rightarrow \infty\right)$, where $P_{m}, m=0, \ldots, k$, are certain polynomials and $\phi_{0, \mathscr{M}}$ is the normal density with zero mean and the covariance matrix $\mathscr{M}$ (see Assumption (i)).

Let $q_{n}$ denote the Fourier transform of $D_{j} h_{n}$, where

$$
h_{n}(x):=g_{n}(x)-\sum_{m=0}^{k} n^{-m / 2} P_{m}(x) \phi_{0, \mathscr{M}}(x) .
$$

Using (A.15), (A.16), and in [25, Lemma 7.2, Ch. 2], we can obtain that for each fixed $j$

$$
q_{n}(t)=t_{j}\left[\phi_{n}(t)-\sum_{m=0}^{k} n^{-m / 2} \widehat{P}_{m}(t) \exp \left(-\frac{1}{2}\langle t, \mathscr{M} t\rangle\right)\right],
$$

with certain polynomials $\widehat{P}_{m}$.

By arguments similar to those given in [24], it follows from (A.18) that there exist constants $\beta>0, c<\infty$ such that

$$
\int_{|t| \leq \beta \sqrt{n}}\left|q_{n}(t)\right| d t \leq \frac{c}{n^{k / 2}} \quad(n \geq l) .
$$

On the other hand, to prove that

$$
\int_{|t|>\beta \sqrt{n}}\left|q_{n}(t)\right|=O\left(\frac{1}{n^{k / 2}}\right), \quad \text { as } n \longrightarrow \infty,
$$

it is sufficient to show that

$$
\int_{|t|>\gamma \sqrt{n}}\left|t_{j}\right|\left|\phi_{n}(t)\right| d t=O\left(\frac{1}{n^{k / 2}}\right), \quad \text { as } n \longrightarrow \infty .
$$


But the last equality follows from (A.12) and the fact that

$$
\sup _{|t|>\gamma \sqrt{n}}\left|\phi_{1}\left(\frac{t}{\sqrt{n}}\right)\right| \leq \kappa<1
$$

Expressing $D_{j} h_{n}$ in terms of $q_{n}$ (as an inverse Fourier transform), and using (A.16)-(A.20) we can establish that there exist a constant $\bar{c}$ and polynomials $\widetilde{P}_{m}(x), m=0, \ldots, k$ such that for each $j \in\{1, \ldots, k\}$,

$$
\sup _{x \in \mathbb{R}^{k}}\left|D_{j} g_{n}(x)-\sum_{m=0}^{k} n^{-m / 2} \widetilde{P}_{m}(x) \phi_{0, \mathscr{M}}(x)\right| \leq \frac{\bar{c}}{n^{k / 2}},
$$

$n=l, l+1, \ldots$

The next step is to find for each $j \in\{1, \ldots, k\}$ an upper bound for $\left\|D_{j} g_{n}\right\|_{\mathbb{R}_{1}\left(\mathbb{R}^{k}\right)}$ which does not depend on $n \geq s$.

For $n>s, g_{n}(x)=n^{k / 2} f_{n}(\sqrt{n} x)$ and $f_{n}(x)=\int_{\mathbb{R}^{k}} f_{s}(x-$ t) $f_{n-s(t)} d t$. Thus, using Assumptions (ii), (iii), and the corresponding theorems in [23, Appendix A], we get

$$
D_{j} f_{n}(x)=\int_{\mathbb{R}^{k}} D_{j} f_{s}(x-t) f_{n-s}(t) d t
$$

We have

$$
\left\|D_{j} g_{n}\right\|_{\mathbb{R}_{1}\left(\mathbb{R}^{k}\right)} \leq \int_{|x| \leq 2 \sqrt{n} \alpha}\left|D_{j} g_{n}\right| d x+\int_{|x|>2 \sqrt{n} \alpha}\left|D_{j} g_{n}\right| d x,
$$

where $\alpha$ is the constant from Assumption (iv). The first summands on the right-hand side of (A.25) are uniformly bounded in $n \geq l$ due to (A.23). To bound the second terms in (A.25) we write (see (A.24))

$$
\begin{aligned}
& \int_{|x|>2 \sqrt{n} \alpha}\left|D_{j} g_{n}\right| d x \\
& \quad=n^{1 / 2} \int_{|z|>2 \alpha n}\left|D_{j} f_{n}(z)\right| d z \\
& \leq n^{1 / 2} \int_{|z|>2 \alpha n} d z \int_{\mathbb{R}^{k}}\left|D_{j} f_{s}(z-t)\right| f_{n-s}(t) d t=: n^{1 / 2} I_{n} .
\end{aligned}
$$

Now

$$
\begin{aligned}
I_{n}= & \int_{|z|>2 \alpha n} d z \int_{|t| \leq \alpha n}\left|D_{j} f_{s}(z-t)\right| f_{n-s}(t) d t \\
& +\int_{|z|>2 \alpha n} d z \int_{|t|>\alpha n}\left|D_{j} f_{s}(z-t)\right| f_{n-s}(t) d t .
\end{aligned}
$$

From $|z|>2 \alpha n$ and $|t| \leq \alpha n$ it follows that $|z-t|>\alpha n$. Thus, the first terms on the right-hand side of (A.27) are less than $c^{\prime} / n^{1 / 2}$ due to Assumption (iv). Applying the Fubini theorem and using the fact of integrability of $D_{j} f_{s}$ we see that the second summand in (A.27) is bounded by const $P\left(\left|\sum_{i=1}^{n-s} X_{i}\right|>\alpha n\right)$, which is $O(1 / n)$ by the Chebyshev and Rosenthal inequalities.
Exploiting Lemmas A.1 and A.2, the rest of the proof of the Theorem 5 in Section 3 is carried out exactly as the proof in one-dimensional case given in [20]. The "ideality properties" of the metric $\zeta_{2}$,

$$
\zeta_{2}\left(a \sum_{i=1}^{n} X_{i}, a \sum_{i=1}^{n} \widetilde{X}_{i}\right) \leq a^{2} \sum_{i=1}^{n} \zeta_{2}\left(X_{i}, \widetilde{X}_{i}\right)
$$

used there hold true for random vectors (see, e.g., [14]). The general version of inequality (3.15) in [20] which relates the Kantorovich and the total variation metrics is proved in [13, page 89]. Note that the proof presented in [20] uses the so-called convolution approach (see, e.g., [26]) and induction arguments. This method has been widely used to estimate rates of convergence in multidimensional Central Limit Theorems (see, e.g., $[13,14,21,26])$.

\section{Acknowledgments}

The authors thank the National System of Investigators (SNI) of CONACYT, Mexico for the partial support of this work. The authors are grateful to the anonymous referee for his valuable suggestions on the improvement of an earlier version of this paper.

\section{References}

[1] F. R. Hampel, "A general qualitative definition of robustness," Annals of Mathematical Statistics, vol. 42, pp. 1887-1896, 1971.

[2] F. R. Hampel, E. M. Ronchetti, P. J. Rousseeuw, and W. A. Stahel, Robust Statistics: The Approach Based on Inuence Functions, Wiley, New York, NY, USA, 1986.

[3] P. J. Huber, Robust Statistics, Wiley, New York, NY, USA, 1981.

[4] J. Jurečková and P. K. Sen, Robust Statistical Procedures. Asymptotics and Interrelations, Wiley, New York, NY, USA, 1996.

[5] X. He and G. Wang, "Qualitative robustness of $S^{*}$-estimators of multivariate location and dispersionl," Statistica Neerlandica, vol. 51, no. 3, pp. 257-268, 1997.

[6] L. Davies, "An efficient Fréchet differentiable high breakdown multivariate location and dispersion estimator," Journal of Multivariate Analysis, vol. 40, no. 2, pp. 311-327, 1992.

[7] D. L. Donoho and R. C. Liu, "The "automatic" robustness of minimum distance functionals," Annals of Statistics, vol. 16, pp. 552-586, 1988.

[8] X. He and D. G. Simpson, "Lower bounds for contamination bies: globally minimax versus locally linear estimation," The Annals of Statistics, vol. 21, pp. 314-337, 1993.

[9] A. Y. Kharin and P. A. Shlyk, "Robust multivariate Bayesian forecasting under functional distortions in the $\chi^{2}$-metric," Journal of Statistical Planning and Inference, vol. 139, no. 11, pp. 3842-3846, 2009.

[10] A. Cuevas, "Qualitative robustness in abstract inference," Journal of Statistical Planning and Inference, vol. 18, no. 3, pp. 277289, 1988.

[11] P. J. Huber, "Robust estimation of a location parameter," Annals of Mathematical Statistics, vol. 35, pp. 73-101, 1964.

[12] R. Zielinski, "Robustness of sample mean and sample median under restrictions on outliers," Zastosowania MatematykiApplicationes Mathematicae, vol. 19, pp. 239-240, 1987. 
[13] S. T. Rachev and L. R. Rüshendorf, Mass Transportation Problems, vol. II: Applications, Springer, New York, NY, USA, 1998.

[14] V. Zolotarev, "Probability metrics," Theory of Probability and Its Applications, vol. 28, pp. 264-287, 1983.

[15] C. G. Small, “A survey of multidimensional medians," International Statistical Review, vol. 58, pp. 263-277, 1990.

[16] J. H. B. Kemperman, “The median of a finite measure on Banach space," in Statistical Data Analysis Based on the $\mathrm{L}_{1}$-Norm and Related Methods, Y. Dodge, Ed., pp. 217-230, Elsevier Science Publishers, North Holland, 1987.

[17] D. Gervini, "Robust functional estimation using the median and spherical principal components," Biometrika, vol. 95, no. 3, pp. 587-600, 2008.

[18] P. Chandhuri, "Multivariate location estimation using extension of $R$-estimates through $U$-statistics type approach," Annals of Statistics, vol. 30, pp. 897-916, 1992.

[19] I. Mizera, "Qualitative robustness and weak continuity: the extreme function? Nonparametrics and robustness in modern statistical inference and time series analysis," in A Festschrift in Honor of Professor Jana Jurečková Institute of Mathematical Statistics Collections, J. Antoch, M. Hušková, and P. K. Sen, Eds., vol. 7, pp. 169-181, Institute of Mathematical Statistics, Beachwood, Ohio, USA, 2010.

[20] E. Gordienko and A. Novikov, "Probability metrics and robustness: is the sample median more robust than the sample mean?" in Proceedings of the Joint Session of 7th Prague Symposium on Asymptotic Statistics and 15th Prague Conference on Information Theory, Statistics Decision Functions, pp. 374-386, Charles University in Prague, Prague, Czech Republic, 2006.

[21] S. T. Rachev, Probability Metrics and the Stability of Stochastic Models, Wiley, Chichester, UK, 2009.

[22] A. W. Van der Vaart, Asymptotic Statistics, Cambridge University Press, Cambridge, UK, 1998.

[23] R. M. Dudley, Uniform Central Limit Theorem, Cambridge University Press, Cambridge, UK, 1999.

[24] E. Gordienko, "Comparing the distributions of sums of independent random vectors," Kybernetika, vol. 41, no. 4, pp. 519529, 2005.

[25] R. N. Bhattacharya and R. Ranga Rao, Normal Approximation and Asymptotic Expansions, Wiley, New York, NY, USA, 1976.

[26] V. V. Senatov, "Uniform estimates of the rate of convergence in the multidimensional central limit theorem," Theory of Probability and Its Applications, vol. 25, pp. 745-759, 1980. 


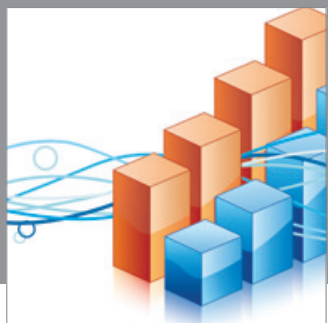

Advances in

Operations Research

mansans

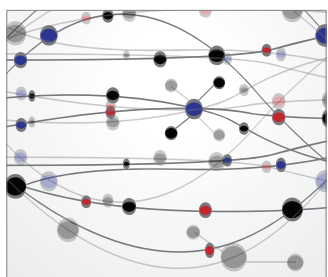

The Scientific World Journal
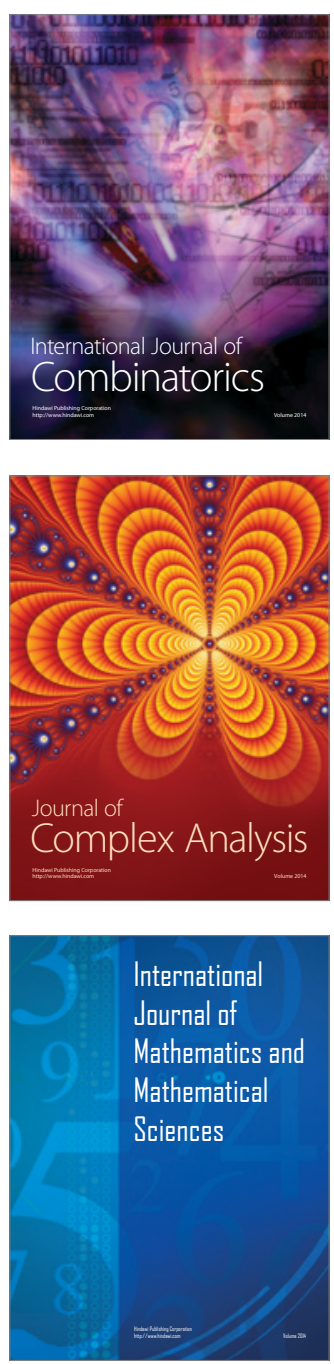
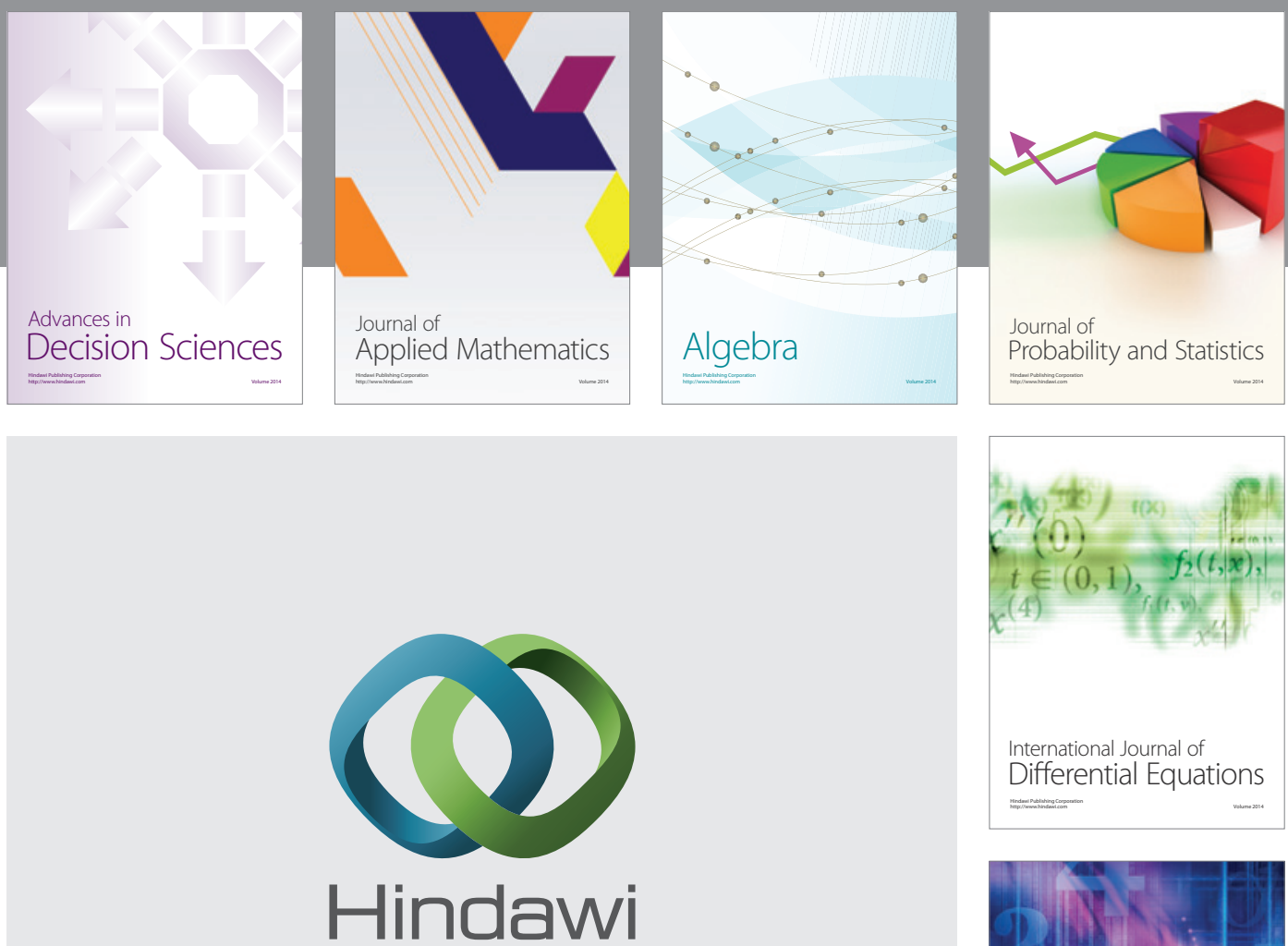

Submit your manuscripts at http://www.hindawi.com
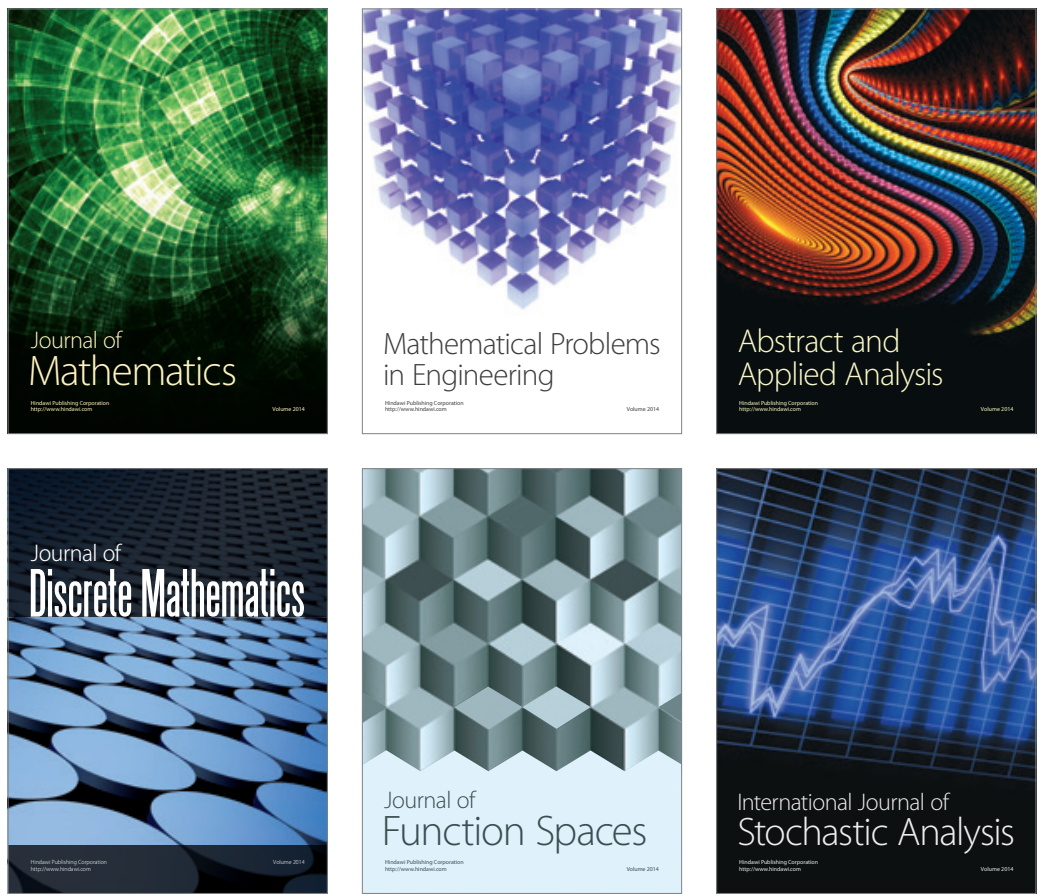

Journal of

Function Spaces

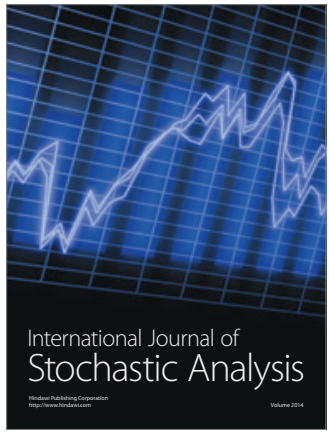

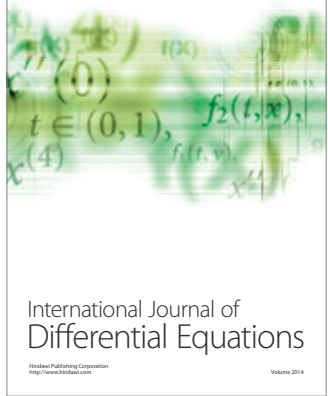
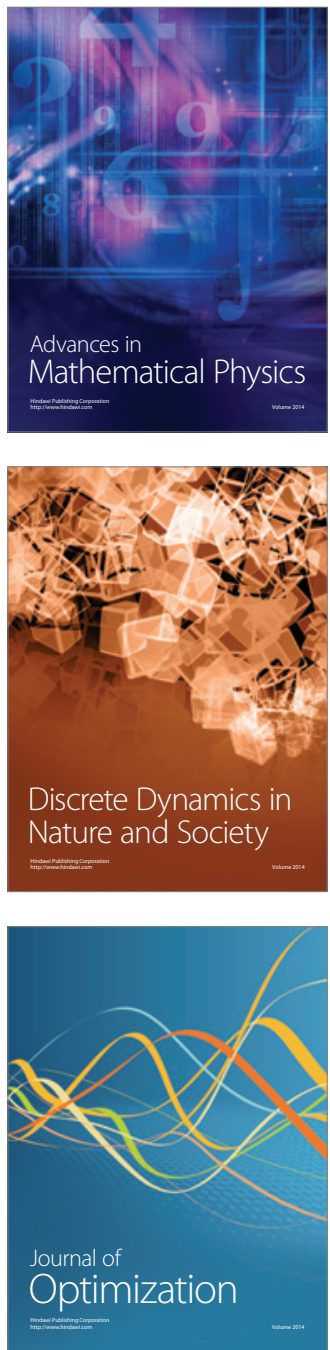